\title{
Descripción de la dieta de una población de Oophaga histrionica (Athesphatanura: Dendrobatidae) en un enclave seco del Valle del Cauca, Colombia
}

\section{Description of the diet of a population of Oophaga histrionica (Athesphatanura: Dendrobatidae) in a dry spot in the Cauca Valley, Colombia}

\author{
Daniel Osorio-Domínguez ${ }^{1,2}$, Leonor Valenzuela ${ }^{3}$, \\ Christian Bermúdez-Rivas ${ }^{4}$, Santiago Castaño ${ }^{5}$
}

\begin{abstract}
Resumen
Se describe la dieta de Oophaga histrionica (Berthold, 1845) basados en el contenido estomacal de 46 individuos colectados en un enclave seco de la Cordillera Occidental de Colombia. Se registran 2322 ítem de presas, clasificados en 21 grupos taxonómicos. Las hormigas y los ácaros fueron el ítem de presa con mayor índice de importancia $\left(I_{i}\right)$ con 44.32 y 12.13 respectivamente. Como las hormigas son el ítem de presa más importante para estas ranas y están relacionadas con los precursores de alcaloides en los dendrobatidos, identificamos las hormigas hasta el nivel de género. Los géneros con mayores índices de importancia fueron solenopsis (51.89), Monomorium (11.55) y Wasmania (11.2), los cuales son reconocidos por producir alcaloides piperidínicos. Por otra parte, aunque la amplitud del nicho de $O$. histrionica no es muy amplia, si es mayor que en otras especies estrechamente relacionadas con los cuales se comparó.
\end{abstract}

Palabras clave: Alcaloides lipofílicos, Amplitud de nicho, Contenidos estomacales, Dieta, Hormigas.

\begin{abstract}
We describe the diet of Oophaga histrionica (Berthold, 1845) based on the stomach contents of 46 individuals collected in a dry spot of the Cordillera Occidental in Colombia. We recorded 2322 prey item, classified in 21 taxonomic groups. Ants and mites were the prey item with the highest importance index $\left(I_{i}\right)$ with 44.32 and 12.13 respectively. Since ants are the most important prey item for these frogs and are related to the precursors of alkaloids in the dendrobatids, we identified the ants to genus level. The genera of ants with higher levels of importance are Solenopsis (51.89), Monomorium (11.55) and Wasmania (11.2), which are known to produce piperidine alkaloids. Moreover, although the niche breadth of $O$. histrionica is not extensive, if greater than in other closely related species with which it was compared.
\end{abstract}

Keywords: Ants, Diet, Food, Lipophilic alkaloids, Niche breadth, Stomach content.

\section{Introducción}

Las ranas de la familia Dendrobatidae se caracterizan por sus colores aposemáticos (con excepciones en los géneros Hyloxalus, Silverstoneia, Colostethus y toda la subfamilia Aromobatinae), sus hábitos diurnos y su actividad terrestre, muy ligada con la hojarasca (Vitt y Caldwell 2009); en este hábitat, la disponibilidad de ártropodos es alta, debido a los procesos ecológicos ligados con la descomposición de la materia orgánica que ellos llevan a cabo ahí (Donnelly 1991, Toft 1995). Por esta ra-

Grupo de Ecología Animal, Universidad del Valle, Cali, Colombia.

Departamento de Ciencias Ecológicas, Universidad de Chile, Santiago, Chile. e-mail: danielosorio77@gmail.com

Departamento de Ecología, Pontificia Universidad Católica de Chile, Santiago, Chile.

Grupo de Investigación en Hormigas, Universidad del Valle, Cali, Colombia.

Facultad de Salud, Universidad del Valle, Cali, Colombia.

Fecha recepción: Noviembre 29, $2014 \quad$ Fecha aprobación: Diciembre 22, $2014 \quad$ Editor asociado: Mantilla H. 
zón, los artrópodos se convierten en un recurso alimenticio muy importante para esta familia (Stork y Blackburn 1993). Sin embargo, este tipo de forrajeo expone a estas ranas a una alta tasa de depredación.

Esto ha llevado a que se propongan hipótesis que correlacionan la dieta con la coloración aposemática y la producción de toxinas (Summers y Clough 2001, Santos et al. 2003, Saporito et al. 2009, Darst et al. 2005, Maan y Cummings 2012, Saporito et al. 2012 no está en la lista de ref.). Estudios de dieta realizados para esta familia han registrado que los ítem alimenticios mejor representados son las hormigas, ácaros y escarabajos (Silverstone 1975, Caldwell 1996, Biavati et al. 2004, Valderrama-Vernaza et al. 2009, Mebs et al. 2010), Arce-Domínguez y RengifoMosquera 2013). Sin embargo, algunos investigadores han sugerido que este grupo tiene una dieta especializada en hormigas (Toft 1995, Caldwell 1996, Santos et al. 2003). La relevancia de este hecho, radica en que se tiene conocimiento que algunas hormigas pertenecientes a la subfamilia Myrmicinae, contienen alcaloides piperidínicos similares a los sintetizados por los miembros de esta familia (Spande et al. 1999).

Estudios previos de las ranas de hojarasca, incluidos los dendrobátidos, tienden a enfatizar las amplias características de la dieta, que combina diferentes tipos de presa y reconocen dos gremios alimenticios:

1. Las especies que se alimentan principalmente de hormigas u hormigas y ácaros.

2. Las especies que se alimentan de otros tipos de presa (Toft 1980, 1995).

Esta dicotomía se ha establecido para diferenciar las dietas de las ranas en general entre especialistas y no especialistas, pero no se ha profundizado en la descripción de cada una de estas dietas, perdiéndose información en algunos estudios que se han hecho con estas ranas (Caldwell 1996, Darst et al. 2005, Saporito et al. 2007).

Oophaga histrionica (Berthold 1845), conocida comúnmente como rana venenosa arlequín o rana venenosa común, es una especie con un tamaño entre 28 y $34 \mathrm{~mm}$ (Silverstone 1975), endémica de Colombia, cuya distribución comprende los departamentos de Antioquia, Valle y Chocó, ocupando diferentes pisos altitudinales desde el nivel del mar hasta los 1000 metros (Bolívar et al. 2004). Aunque existen estudios que mencionan los hábitos alimenticios de ciertas especies de dendrobátidos (Donelly 1991, Toft 1995, Caldwell 1996, Biavati et al. 2004, Valderrama-Vernaza et al. 2009), la dieta de $O$. histrionica fue descrita brevemente por Silverstone (1975) a partir de solo ocho individuos. Asimismo, y teniendo en cuenta la relevancia de las hormigas para la dieta de estas especies como apoyo o rechazo de la hipótesis dietética sobre el secuestro de alcaloides (Saporito et al. 2009), es importante adquirir conocimiento más preciso en la dieta de los dendrobátidos. Además, es importante detallar la composición de este ítem en particular, aunque la mayoría de los registros de dieta no lo hacen, porque no todos los géneros de hormigas contienen precursores de alcaloides. Nuestro objetivo en esta nota es describir la dieta para una población de $O$. histrionica ubicada en un enclave seco del departamento del Valle del Cauca, haciendo énfasis en describir los diferentes géneros de hormigas presentes en ella, comparándola con la dieta descrita para algunas especies emparentadas. Una mejor comprensión de la dieta en este grupo proporcionará mayor información para llegar a entender la evolución de la dieta y su relación con la producción de alcaloides en este grupo de anfibios.

\section{Metodología}

Área de estudio. Esta investigación se llevó a cabo en el caserío El Naranjo, municipio de Dagua, departamento del Valle del Cauca $\left(3.780^{\circ} \mathrm{N},-76.719^{\circ}\right.$ O). La localidad es un enclave de bosque seco en la Cordillera Occidental, en el departamento del Valle del Cauca. Es una zona de transición entre el bosque seco tropical y la selva húmeda de la vertiente Pacífico del departamento. Este sitio se encuentra en un pequeño cañón formado por una quebrada, donde las paredes se utilizan como cantera de piedra para mampostería. La mayoría de las ranas se encuentran entre las piedras del suelo y la hojarasca, agrupadas en unos cientos de metros cuadrados.

Métodos. Los especímenes se colectaron entre los meses de febrero y septiembre del año 2002, durante las horas de mayor forrajeo (entre 9:00 y las 11:00 horas). Los individuos colectados se depositaron en bolsas plásticas que eran inmediatamente almacenadas en una nevera portátil con hielo seco 
con el objetivo de detener el proceso de digestión y así evitar la pérdida de información (Caldwell 1996, Parmelee 1999).

Se analizaron 46 especímenes adultos de $O$. histrionica. Cada uno de los individuos se evisceró en la parte ventral derecha para extraer todo el tracto digestivo (intestino y estómago), ya que se ha demostrado que al trabajar solo con los contenidos estomacales se pierde información, debido a que las presas pequeñas pasan más rápido a la porción intestinal, encontrando solo las presas de mayor tamaño en el estómago (Schoener 1989). Estos contenidos estomacales se conservaron en alcohol etílico al $70 \%$. Las presas se identificaron hasta la menor categoría taxonómica posible usando como referencia Johnson y Triplehorn (2004) y Resh y Cardé (2009). Para la familia Formicidae las presas se identificaron hasta género en la mayoría de los casos, utilizando como referencia a Fernández (2003).

Mediante conteo se estableció el número de presas en cada uno de los estómagos. El volumen aproximado de cada presa se calculó utilizando la fórmula volumétrica del esferoide:

$$
V=\frac{4 \pi}{3}\left(\frac{\text { Largo }}{2}\right)\left(\frac{\text { Amplitud }}{2}\right)
$$

Se midió el largo (sin tener en cuenta antenas, mandíbulas, extremidades u ovopositores) y el ancho (en el punto medio del cuerpo) con un calibrador digital (precisión de $0.01 \mathrm{~mm}$ ) de cada presa completa encontrada. Además, se calculó el índice de importancia de cada ítem (I), para reducir la mala interpretación de la significancia de un grupo sistemático con respecto a los demás:

$$
\mathrm{I}=\frac{N \%+V \%}{2}
$$

donde $N \%=$ porcentaje numérico y $V \%=$ porcentaje volumétrico (Obrtel y Holisová 1974).

Mediante una prueba $X^{2}$ se comparó si los porcentajes volumétricos de hormigas (por ser los más representativos) encontrados en $O$. histrionica, difieren de los porcentajes volumétricos registrados para otras especies de dendrobatidos registrados en la literatura. Asimismo, para comprobar si la ampli- tud de nicho de $O$. histrionica se ajusta con las registradas para otras especies emparentadas, calculamos la amplitud de nicho (B) a partir de la ecuación invertida de Simpson:

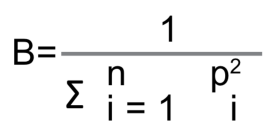

donde $i$ es la categoría del recurso, $p$ es la proporción del recurso de la categoría $i$, y $n$ es el número total de categorías de presa (Pianka 1986).

\section{Resultados}

Se identificaron y se clasificaron 2.322 presas en 22 categorías (Figura 1). Ninguno de los especímenes analizados tenía el sistema digestivo vacío y el número promedio de presas encontrado fue de $47.47 \pm 33.73(\mathrm{~N}=46)$. El índice de importancia (I) muestra que los ítems alimenticios más significativos para esta población fueron las hormigas, las larvas acuáticas de dípteros, los ácaros y los coleópteros (Tabla 1). Las hormigas obtuvieron los más altos valores para las tres variables que el índice de importancia tiene en cuenta. La diferencia en importancia entre los coleópteros, los ácaros y las larvas de díptera, radica fundamentalmente en el número de especímenes capturados, donde las larvas y los ácaros presentan los más altos porcentajes. En cuanto a los volúmenes ocupados, los coleópteros son el segundo ítem que más alto valor tiene.

Se logró un nivel alto de resolución taxonómica a nivel de género para las hormigas (93.1\%). De los 13 géneros hallados, Solenopsis, Monomorium y Wasmania, son los de mayor aporte tanto numérico $(84.2 \%)$ como volumétrico $(65.1 \%)$. Los géneros restantes se encontraron por debajo del $10 \%$ en ambos aportes (Tabla 2). El porcentaje volumétrico de hormigas consumido por $O$. histrionica, no difiere estadísticamente del porcentaje volumétrico registrado para otras especies de la familia Dendrobatidae $\left(X^{2}=8.4741, \mathrm{p}=0.2926\right)$. En cuanto a la amplitud de nicho, puede observarse que el valor hallado para $O$. histrionica es intermedio en comparación con las especies restantes, sin alcanzar los valores de especies pertenecientes al género Allobates (Tabla 3). 


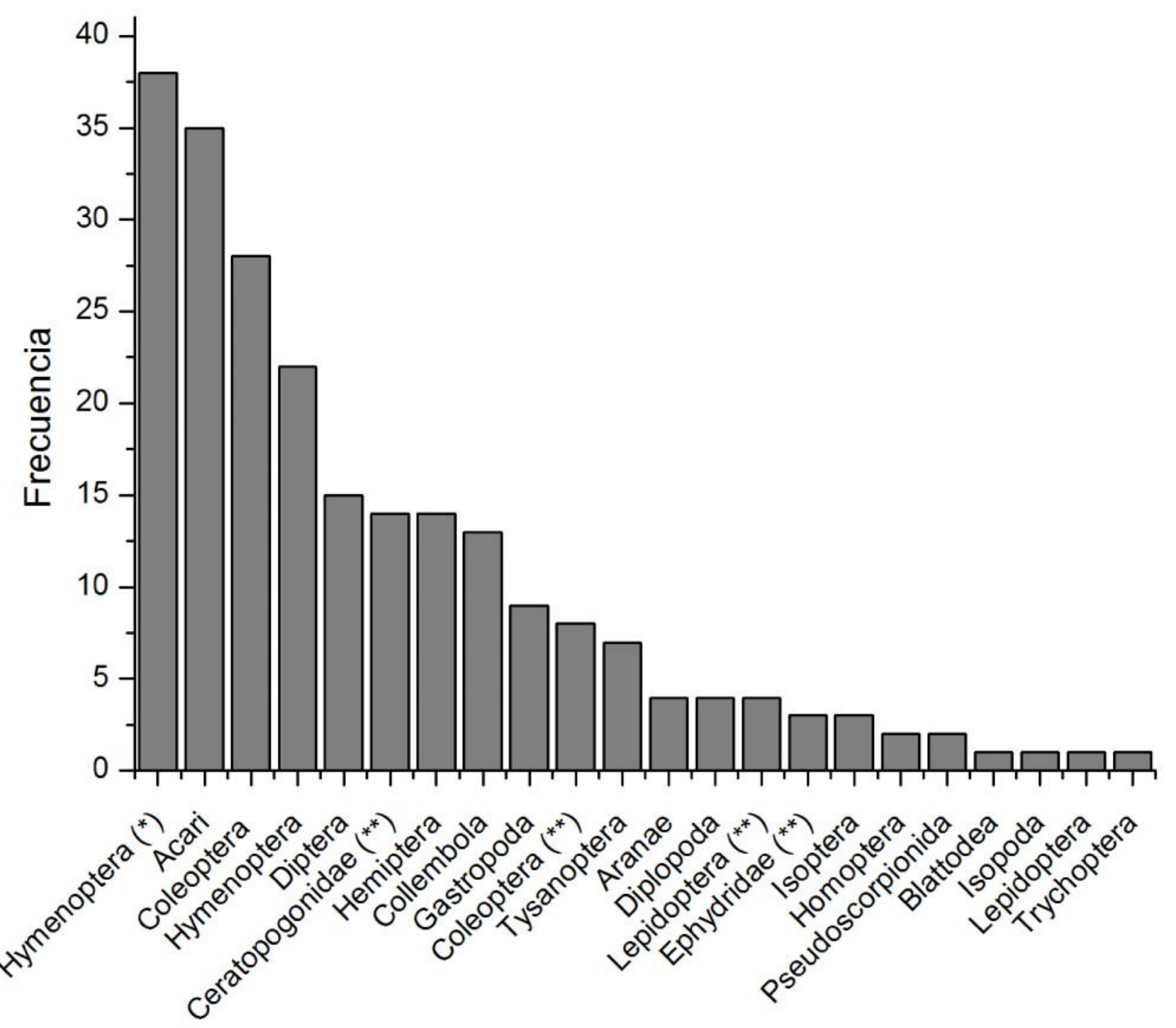

Figura 1. Categorías de presa y su frecuencia encontradas en el sistema digestivo de $O$. histrionica.

${ }^{*}$ Himenópteros pertenecientes a la familia Formicidae, ${ }^{* *}$ Larvas.

\section{Discusión}

Al hacer una comparación general de la dieta de $O$. histrionica con la registrada para otras especies de la familia Dendrobatidae, se encontró que hay consistencia con el patrón, cuyo ítem dietético más importante son las hormigas (Darst et al.2005). Sin embargo, llama la atención que el segundo ítem alimenticio con mayor índice de importancia son las larvas acuáticas de dípteros como los ceratopogónidos. Lo anterior hace suponer que $O$. histrionica, no solo se alimenta de pequeños artrópodos de hoja- rasca, sino que también forrajea en pequeñas charcas formadas en hojas, cavidades de troncos y en las axilas de algunas plantas como aráceas y bromelias, los cuales son lugares habitualmente utilizados por estas ranas para depositar sus renacuajos, donde completan su desarrollo (Summers y McKeon 2004).

La amplitud de nicho y el índice de importancia (I) indicaron que $O$. histrionica es una especie especializada en hormigas. Sin embargo, esta amplitud de nicho es mayor que la reportada para otras especies consideradas especialistas en hormigas (Caldwell 1996, Darst et al. 2005). Asimismo, el porcentaje volumétrico de hormigas a pesar de no 
Tabla 1. Dieta de O. histrionica en una población de un enclave seco en el Valle del Cauca

\begin{tabular}{llllllll}
\hline Categoría de presa & $\mathbf{F}$ & $\mathbf{F} \%$ & $\mathbf{N}$ & $\mathbf{N} \%$ & $\mathbf{V}$ & $\mathbf{V} \%$ & $\mathbf{I}_{\boldsymbol{i}}$ \\
\hline Hymenoptera (Formicidae) & 38 & 82,61 & 1075 & 46,29 & 175,72 & 40,35 & 44,32 \\
Acari & 35 & 76,09 & 381 & 16,41 & 15,62 & 3,59 & 12,13 \\
Coleoptera & 28 & 60,87 & 67 & 2,89 & 88,15 & 20,23 & 8,66 \\
Collembola & 13 & 28,26 & 125 & 5,38 & 12,00 & 2,76 & 4,51 \\
Hymenoptera (no Formicidae) & 22 & 47,83 & 77 & 3,31 & 13,46 & 3,08 & 3,24 \\
Hemiptera & 14 & 30,43 & 35 & 1,51 & 27,85 & 6,40 & 3,13 \\
Gastropoda & 9 & 19,57 & 19 & 0,82 & 26,11 & 5,99 & 2,54 \\
Isoptera & 3 & 6,52 & 15 & 0,65 & 13,37 & 3,07 & 1,45 \\
Diptera & 15 & 32,61 & 24 & 1,03 & 7,61 & 1,75 & 1,27 \\
Homoptera & 2 & 4,35 & 12 & 0,52 & 10,60 & 2,43 & 1,16 \\
Blattodea & 1 & 2,17 & 1 & 0,04 & 9,76 & 2,24 & 0,78 \\
Diplopoda & 4 & 8,70 & 4 & 0,17 & 5,50 & 1,26 & 0,54 \\
Aranae & 4 & 8,70 & 6 & 0,26 & 3,37 & 0,77 & 0,43 \\
Thysanoptera & 7 & 15,22 & 8 & 0,34 & 1,23 & 0,28 & 0,32 \\
Lepidoptera & 1 & 2,17 & 1 & 0,04 & 1,53 & 0,35 & 0,15 \\
Trychoptera & 1 & 2,17 & 1 & 0,04 & 1,64 & 0,38 & 0,15 \\
Pseudoscorpionida & 2 & 4,35 & 2 & 0,09 & 0,72 & 0,17 & 0,11 \\
Isopoda & 1 & 2,17 & 1 & 0,04 & 0,14 & 0,03 & 0,04 \\
Larvas & 14 & 30,43 & 449 & 19,34 & 13,83 & 3,76 & 14,14 \\
Diptera & 4 & 8,70 & 7 & 0,30 & 4,08 & 0,94 & 0,51 \\
Lepidoptera & 8 & 17,39 & 12 & 0,52 & 0,73 & 0,17 & 0,40 \\
Coleoptera & & & & & & & \\
\hline
\end{tabular}

$\mathrm{F}=$ frecuencia de cada ítem, $\mathrm{F} \%=$ porcentaje de $\mathrm{F}, \mathrm{N}=$ número de presas de cada ítem, $\mathrm{N} \%=$ porcentaje de $\mathrm{N}, \mathrm{V}=\mathrm{volumen}$ de las presas, $\mathrm{V} \%=$ Porcentaje de $\mathrm{V}, \mathrm{I}=$ Índice de importancia

Tabla 2. Géneros de las hormigas que componen la dieta de $O$. histrionica

\begin{tabular}{lllllll}
\hline Categoría de presa & $\mathbf{F}$ & $\mathbf{N}$ & $\mathbf{N} \%$ & $\mathbf{V}$ & $\mathbf{V} \%$ & $\mathbf{I}_{\mathbf{i}}$ \\
\hline Solenopsis & 26 & 569 & 52,93 & 89,33 & 50,84 & 51,89 \\
Monomorium & 14 & 192 & 17,86 & 9,22 & 5,25 & 11,55 \\
Wasmannia & 14 & 144 & 13,4 & 15,84 & 9,02 & 11,21 \\
Pheidole & 2 & 14 & 1,3 & 13,4 & 7,63 & 4,46 \\
Atta & 3 & 4 & 0,37 & 8,48 & 4,83 & 2,6 \\
Neivamyrnex & 2 & 26 & 2,42 & 4,68 & 2,66 & 2,54 \\
Gnamptogenys & 1 & 13 & 1,21 & 4,49 & 2,55 & 1,88 \\
Trachymyrmex & 2 & 15 & 1,4 & 3,3 & 1,88 & 1,64 \\
Odontomachus & 4 & 4 & 0,37 & 1,73 & 0,98 & 0,68 \\
Acanthognatus & 6 & 10 & 0,93 & 0,54 & 0,31 & 0,62 \\
Cyphomyrmex & 6 & 6 & 0,56 & 0,97 & 0,55 & 0,55 \\
Pachycondyla & 1 & 3 & 0,28 & 0,52 & 0,3 & 0,29 \\
Crematogaster & 1 & 1 & 0,09 & 0,06 & 0,03 & 0,06 \\
Myrmicinae alados & 4 & 16 & 1,49 & 15,68 & 8,92 & 5,21 \\
Formicinae & 4 & 58 & 5,4 & 7,48 & 4,26 & 4,83 \\
\hline
\end{tabular}

$\mathrm{F}=$ frecuencia de cada ítem, $\mathrm{F} \%=$ porcentaje de $\mathrm{F}, \mathrm{N}=$ número de presas de cada ítem, $\mathrm{N} \%=$ porcentaje de $\mathrm{N}, \mathrm{V}=$ volumen de las presas, $\mathrm{V} \%=$ Porcentaje de $\mathrm{V}, \mathrm{I}_{\mathrm{i}}=$ índice de importancia

presentar diferencias significativas entre especies, es menor que el registrado en especies más estrechamente emparentadas como D. auratus y $O$. pumilio
(Darst et al.2005). El número restante de presas tampoco se diferenció de las reportadas para otras especies forrajeadoras de la hojarasca y el número de 
Tabla 3. Tamaños de muestra, número promedio de presas por individuo, porcentaje numérico y volumétrico de hormigas dentro de la dieta por individuo y amplitud de nicho (B) para especies de dendrobátidos

\begin{tabular}{|c|c|c|c|c|c|c|c|}
\hline \multirow[t]{2}{*}{ Especie } & \multirow[t]{2}{*}{ Fuente } & \multirow[t]{2}{*}{$\mathbf{N}^{\circ}$} & \multirow{2}{*}{$\begin{array}{l}\text { Promedio } \\
\text { de presas }\end{array}$} & \multirow{2}{*}{$\begin{array}{c}\% \text { numérico } \\
\text { hormigas }\end{array}$} & \multirow{2}{*}{$\begin{array}{c}\% \text { volumétrico } \\
\text { hormigas }\end{array}$} & \multicolumn{2}{|c|}{ B } \\
\hline & & & & & & numérico & volumétrico \\
\hline O. pumilio & Caldwell & 33 & $84,4 \pm 9,3$ & 27 & 50 & 2,25 & 2,75 \\
\hline D. auratus & Caldwell & 23 & $186,5 \pm 24,0$ & 63 & 73 & 2,03 & 1,86 \\
\hline O. histrionica & & 46 & $47,47 \pm 33,73$ & 44 & 40 & 3,70 & 4,58 \\
\hline H. sauli & Darst et al. & 9 & $5,3 \pm 1,6$ & 60 & 53 & 2,71 & 3,09 \\
\hline A. talamancae & Caldwell & 19 & $13,9 \pm 2,3$ & 21 & 16 & 7,18 & 11,94 \\
\hline A. zaparo & Darst et al. & 20 & $9,7 \pm 1,4$ & 26 & 4 & 8,31 & 8,14 \\
\hline \multirow[t]{2}{*}{ R. virolinensis } & Valderrama- & & & & & & \\
\hline & Vernaza et al. & 165 & $56 \pm 37,64$ & 4 & 26 & 1,45 & 6,78 \\
\hline A. flavopicta & Biavati et al. & 612 & $13,04 \pm 17,51$ & 47 & 12 & 3,06 & 2,61 \\
\hline
\end{tabular}

ítems no se diferencia del resto de dendrobátidos. Además, el incluir otras presas estaría relacionado con el balance de nutrientes, como se lo sugirieron Donnelly (1991) y Caldwell (1996) para otras especies del género Oophaga, donde los rangos secundarios para las presas comprenden entre el $20 \%$ y el $27 \%$ de la dieta.

La especialización en el consumo de hormigas por parte de algunas especies perteneciente a la familia Dendrobatidae ha sido asociada con el secuestro y síntesis de alcaloides (Donnelly 1991, Daly et al. 1994, Daly et al. 1997, Darst et al. 2005). La caracterización detallada de las hormigas encontradas en la dieta de $O$. histrionica da una idea de cuáles serían los géneros más relevantes como fuentes para la síntesis de alcaloides lipofílicos (p.e. piperidinas y decahidroquinolinas) de la misma clase estructural identificados en otros dendrobátidos (Spande et al. 1999). Es el caso del genero Solenopsis, el cual estaría aportando decahydroquinolinas, componente principal de las histrionicotoxinas características del genero Oophaga (Spande et al. 1999). Asimismo, se encuentra como segundo ítem en importancia dentro de las hormigas el género Monomorium, donde Daly et al. (1994) registraron precursores de pyrrolidinas como las detectadas en especímenes de D. auratus.

Finalmente, con un índice de importancia parecido al del género Monomorium, aparece el género Wasmania, el cual a pesar de no ser registrado en la literatura como precursor de alcaloides, sí se tiene conocimiento que otros géneros pertenecientes a la subfamilia Myrmicinae, poseen pyrrolidinas en sus venenos (Jones et al. 1999).

\section{Conclusiones}

La dieta concuerda en términos generales con lo registrado en la bibliografía para otras especies de la familia. Sin embargo, la amplitud de nicho es mayor si se compara especies cercanas como $O$. pumilio y su porcentaje volumétrico de hormigas es menor. También, la importancia de hormigas del género Solenopsi, como de ácaros en la dieta coincide con la hipótesis alimentaria, la cual relacionan la dieta con la producción de toxinas en la piel.

\section{Agradecimientos}

Nosotros deseamos agradecer a Leonardo Rivera por la ayuda en la identificación de las hormigas. Helberg Asencio, Julián Velasco, Vladimir Rojas y Carlos A. Saavedra por la ayuda en campo. También agradecemos a la Corporación Regional del Valle del Cauca (CVC) por otorgar los permisos de colecta científica y a la Universidad del Valle por su apoyo institucional, académico y científico. Finalmente agradecemos a los revisores anónimos por sus sugerencias.

\section{Literatura citada}

Arce-Domínguez F, Rengifo-Mosquera JT. 2013. Dieta de Phyllobates aurotaenia y Oophaga histrionica (Anura: Dendrobatidae) en el municipio de Alto Baudó, Chocó, Colombia. Acta Zool Mex. 29: 255-68.

Biavati GM, Wiederehecker HC, Colli GR. 2004. Diet of Epipedobates flavopictus (Anura: Dendrobatidae) in a neotropical savanna. J Hepetol. 38: 510-8. 
Bolivar W, Lötters S, Grant T. 2004. Oophaga histrionica. The IUCN Red List of threatened species. Version 2014. 3 URL disponible en: http://www.iucnredlist.org/ www.iucnredlist.org (Downloaded on 15 January 2015).

Caldwell JP. 1996. The evolution of myrmecophagy and its correlates in poison frog (Family Dendrobatidae).J Zool. (London) 240: 75-101.

Daly JW, Secuda SI, Garrafo HM, Spande TF, Wisnieski A, Cover JF. 1994. An uptakes system for dietary alkaloids in poison frogs (Dendrobatidae). Toxicon. 36: 657-63.

Daly JW, Garrafo MH, Myers CW. 1997. The origin of frog skin alkaloids: an enigma. Pharmaceut News. 4: 9-14.

Darst CR, Menéndez-Guerrero PA, Coloma LA, Canatella DC. 2005. Evolution of dietary specialization and chemical defense in poison frogs (Dendrobatidae): a comparative analysis. Am Natural. 156: 56-69.

Donnelly MA. 1991. Feeding patterns of the strawberry poison frog, Dendrobates pumilio (Anura: Dendrobatidae). Copeia. 3: 723-30.

Fernández F (ed.). 2003. Introducción a las hormigas de la región neotropical. Bogotá: Instituto de Investigación de Recursos Biológicos Alexander von Humboldt; XXVI + $398 \mathrm{p}$.

Johnson NF,Triplehorn CA. 2004. Borror and DeLong's introduction to the study of insects. 7 ed. Boston: Brooks Cole; 888 p.

Jones TH, Wojciechowski TJ, Snelling RR, Torres JA, Chacón P, Devries PJ. 1999. Dialkilpyrroloidinas from the ants Megalomyrmex cyendyra Brandao and $M$. latreillei Emery. Caribbean J Sci. 35 (3-4): 310-1.

Maan EM, Cummings ME. 2012. Poison frog colors are honest signals of toxicity, particularly for bird predators. Am Naturalist. 179: E1-E14.

Mebs D, Jansen M, Köhler G, Pogoda W, Kauert G. 2010. Myrmecophagy and alkaloid sequestration in amphibians: a study on Ameerega picta (Dendrobatidae) and Elachistocleis sp. (Microhylidae) frogs. Salamandra.46: 11-5.

Obrtel R, Holisová V. 1974. Trophic niches of Apodemus flavicollis and Clethrionomys glareolus in lowland forest. Acta Scient Nat Acad Scient Bohem Brno. 8 (7): 1-37.

Parmelee JR. 1999. Trophic ecology of a tropical anuran assemblage. Sci Pap Nat Hist Mus The University of Kansas. 11: 1-59.

Pianka ER. 1986. Ecology and natural history of desert lizards: analyses of the ecological niche and community structure. Princeton: Princeton University Press; 222 p.

Resh VH, Cardé RT. 2009. Encyclopedia of insects. Burlington: Academic Press; 1132 p.

Santos JC, Coloma LA, Canatella DC. 2003. Multiple, recurring origins of aposematism and diet specialization in poison frogs. Proc Nat Acad Sci. USA 100: 12792-7.

Saporito RA, Spande TF, Garrafo HM, Donelly MA. 2009. Arthropod alkaloids in poison frogs: A review of the 'dietary hypothesis'. Heterocycles. 79: 277-97.

Saporito RA, Donnelly MA, Norton RA, Garraffo HM, Spande TF, Daly JW. 2007. Oribatid mites as a major dietary source for alkaloids in poison frogs. Proceed Nat Acad Sci USA. 104: 8885-90.

Schoener TW. 1989. Should hindgut contents be included in lizard dietary compilations? J Herpetol. 23: 455-8.

Silverstone PA. 1975. A revision of the poison-arrow frogs of the genus Dendrobates Wagler. Natural History Museum of Los Angeles County. Sci Bull. 21: 1-55.

Spande TF, Jain P, Garrafo MH, Pannell LK, Yeh HJC, Daly JW, et al. 1999. Ocurrence and significance of decahydroquinolines from dendrobatid poison frogs and myrmicine ants: use of ${ }^{1} \mathrm{H}$ and ${ }^{13} \mathrm{C}$ NMR in their conformational analysis. J Nat Prod. 62: 5-21.

Storke NE, Blackburn TM. 1993. Abundance, body size and biomass of arthropod in tropical forest. Oikos. 67: 4839.

Summers K, Clough ME. 2001. The evolution of coloration and toxicity in the poison frog family (Dendrobatidae). Proc Nat Acad Sci. USA 11: 6227-32.

Summers K, McKeon CS. 2004. The evolutionary ecology of phytotelmata use in neotropical poison frogs. Miscellaneous Publications Museum of Zoology University of Michigan. 193: 55-73.

Toft CA. 1980. Feeding ecology of thirteen syntopic species of anurans in a seasonal tropical environment. Oecologia. 45: $131-41$.

Toft CA. 1995. Evolution of diet specialization in poison-dart frogs (Dendrobatidae). Herpetologica. 51: 202-16.

Valderrama-Vernaza M, Ramírez-Pinilla MP, Serrano-Cardozo VH. 2009. Diet of the andean frog Ranitomeya virolensis (Athesphatanura: Dendrobatidae). J Herpetol. 43: 11423.

Vitt LJ, Caldwell JP. 2009. Herpetology. $3^{\text {rd }}$ ed. Burlington: Academic Press. 713 p. 